\title{
A Review of Multimodal Pragmatics and Translation: A New Modal for Source Text Analysis
}

\author{
$\mathrm{Ma} \mathrm{Xiao}^{1}$, Ye Yanping ${ }^{2}$
}

\author{
${ }^{1}$ School of Foreign Languages and Literature, Wuhan University, China \\ Email: ma.xiao@whu.edu.cn \\ ${ }^{2}$ School of Foreign Languages, Xi' an University of Finance and Economics, China \\ Email: ye.yanping@163.com
}

\begin{abstract}
This paper is a book review of Multimodal Pragmatics and Translation: A New Modal for Source Text Analysis. It first depicts an overview of the whole picture of the book with a brief summary of each chapter, and then discusses how it develops a model for textual analysis of multimodal source texts applicable to translation studies from a pragmatic perspective. Finally, the merits and demerits in this book are pointed out.
\end{abstract}

Keywords - multimodality, ST analysis, pragmatic perspective, translation studies.

\section{INTRODUCTION}

Multimodality has become a focus of interest to academic research in recent years with the rapid advancement of modern technology and emergence of new media. An increasing number of scholars begin to investigate ways of text analysis from a multimodal perspective rather than traditional approaches concentrating on language itself only and are aware of the importance of multimodal analysis in the field of translation studies. This book aims to develop a model for the textual analysis of multimodal source texts (STs) for translation purposes. The author brings together "aspects of pragmatics, studies on multimodality, and translation studies and semiotics" (p.160) under the umbrella of Relevance Theory (Sperber and Wilson, 1986, 1995) on a quest for the meaning-making mechanism of multimodal text analysis that combine images and words. And the well founded analysis and detailed elaboration have proved her great success.

\section{AN OVERVIEW OF THE BOOK}

Chapter 1 serves as somewhat an introduction to the whole book. It begins with a brief discussion of the development of translation studies, pointing out the necessity of "shifting the spotlight from language to a detailed analysis of how a variety of multimodal text types convey meaning" (p. 3). Then it offers an overview of the whole work, indicating the intention of the book is to build a model for the translation-oriented analysis of multimodal ST given the lack of a generally applicable framework. This chapter also outlines what is going to be discussed in the ensuing chapters.

Understanding in detail how signs from different semiotic sources interact to generate meaning is fundamental for a study on multimodality, as it provides a picture of how a multimodal text is organized to convey messages. This is why Chapter 2 reviews the relevant literature on semiotics from the very start. The chapter is also dedicated to a critical discussion on Kress and Van Leeuwen's Multimodal Discourse (2001) and Baldry and Thibault's Multimodal Transcription and Text Analysis (2005) in an attempt to find some 'overriding principles' that govern and guide multimodal meaning generation (p.23). However, their efforts failed and the potential overriding principles have to be sought elsewhere. That's why pragmatics comes to the fore.

Chapter 3 discusses the literature relevant to the understanding of multimodal meaning in context from a pragmatic perspective and how pragmatic approaches can be 
applied to the analysis of multimodal texts for translation purposes. It first reviews the studies on multimodality based on two major pragmatic theories such as Grice's theory of cooperativeness and Sperber and Wilson's Relevance Theory (RT), and then the literature on pragmatics and translation studies is also analyzed so as to build the "link between a pragmatic analysis of multimodal STs and their translation" (p.52). Note that Chapter 3 is in fact a stepping stone to the subsequent chapters.

Chapter 4 is the most important part of this work because it witnesses the development of the new model from its theoretical grounding to its full form. Following Sperber and Wilson's RT, it attempts to develop a translation-oriented model for the systematic analysis of multimodal texts. Dicerto's main sources of inspiration come from Sperber and Wilson's cognitive pragmatic theory, Martinec and Salway's visual-verbal relations, and Pastra's visual-verbal relations with multimodal meaning. The new model proposed generally consists of three dimensions, namely pragmatic analysis based on the distinction between explicit and implicit meaning outlined by Sperber and Wilson, visual-verbal relations and the meaning of individual modes. This three-dimensional view offers a holistic understanding of multimodal texts and their potential translation issues from the most general to the most specific dimension of analysis in multimodal texts in order to achieve the interpretive resemblance (Gutt, 2000) according to the principle of optimal relevance in actual translation scenarios. In Chapter 5, the model proposed is applied to the practical multimodal ST analysis for translation purposes, for which 12 authentic texts are selected to discuss in details. The author first introduces the selection of material, the analytical procedure and the coding system, and then presents the empirical analysis by dividing the multimodal texts into expressive texts, informative texts and operative texts in three sections based on Reiss's text taxonomy. This perhaps becomes the most appealing part of the book for readers, to which one third of the space (60 pages) is devoted.

\section{A CRITICAL REVIEW}

As we know, any model needs an assessment of its applicability and validity. To conclude the book, Chapter 6 provides an assessment to what extent the model has met its initial objectives and offers a few reflections on the work, such as the original goal, the current form of the model, its contributions to the study of multimodality, its limitations and its future applicability within and outside translation studies.

Compared with recent studies in the literature, Dicerto's book has taken extensive study on multimodality, linking visual/verbal relations not only to issues connected to meaning but also to translation matters. The new model for understanding of "how multimodal texts are organized to convey meaning and of what this means when it comes to rendering them into a target text" (p.3) is also unprecedented (Chen, 2019). Moreover, the author develops her ideas in a very logical manner. Each chapter is naturally "on the road" (p.15) to the next, and chapters clearly build upon one another. And the integration of theory and practice is a further manifestation of it. This book also demonstrates the author's objective scientific attitude. For instance, although the author suggests that the model sets out to include provision for the analysis of all types of multimodal STs, the application of the model later exemplified only uses static multimodal texts without including dynamic ones. It could have become a denouncement by some scholars, yet Sara explicitly acknowledged this and offers a convincing explanation early in the introduction. Also, the model seems to have a pervasive application, and it is not proper "for professional translators in their everyday activity" (p.96) because it is too extremely time consuming.

Despite the aforementioned merits, this publication also has its limitations concerning both the content and the design. Evidently the first chapter is an "introductory chapter" (p.4) mapping the author's ideas for the whole work and the structure of the following chapters, thus the title for Chapter 1 should be "introduction" (Sara, 2014) just like her doctoral dissertation (p.1) instead of the current "A New Model Source Text Analysis in Translation", which seems too early for a start. Besides, the model proposed in the book is complete in structure, and it is yet still difficult to operate due to its complexity; it would be better for the author to say a bit more on how to designate the different parts in the multimodal ST (Chen, 2019). For example, why in the "backbone" example, the image and the text are classified into different clusters while in the "the cat in the hat" example, the image and the text are put in the same one 
cluster? As for the design of the book, two things can be improved despite Sara's kind considerations. On one hand, the "CONTENTS" page looks a bit too simple and rough. Wouldn't it be better if the subsections and their pages of each chapter had also been included? In so doing, readers will get a general view of the book quickly by having a glance at this page. On the other hand, the foreword part of the book seems to be missing. A foreword may make the publication seemingly more professional and valuable.

\section{CONCLUDING REMARKS}

To sum up, this work is like a wonderful journey of the editor. If a title were to be given to this review, it would be Sara's Model Hunt: A pragmatic multimodal ST analysis for translation. This journey is accessible, clear-targeted, informative, and fruitful, for which it deserves a strong recommendation to professionals, translation researchers and translation tutors as agreed by the author (p.96). It would also be of interest to language teachers and students who are keen to get updated information on the latest development and innovations in the translation field.

\section{REFERENCES}

[1] Chen, J. R. 2019. Duomotai Yuyongxue Fenxifa Zai Fanyi Yanjiu Zhongde Yingyong [Applying Multimodal Pragmatic Analysis in Translation Studies]: A review on Multimodal Pragmatics and Translation: A New Model for Source Text Analysis. Chinese Translators Journal, 40(1), 111-116.

[2] Dicerto, Sara, 2014. Multimodal Pragmatics: Building A New Model for Source Text Analysis (PhD dissertation). England, University of Surrey.

[3] Gutt, E. A. 2000. Translation and Relevance: Cognition and Context ( $2^{\text {nd }}$ edition), Manchester: St. Jerome.

[4] Sperber, D. \& D. Wilson. 1986/1995. Relevance: Communication and Cognition ( $2^{\text {nd }}$ edition), Oxford: Blackwell. 